\title{
Non-linear relationship between maternal work hours and child body weight: Evidence from the Western Australian Pregnancy Cohort (Raine) Study
}

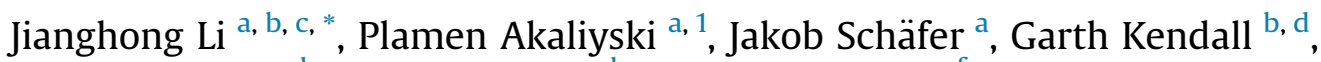 \\ Wendy H. Oddy ${ }^{\mathrm{b}}{ }^{\mathrm{e}}$, Fiona Stanley ${ }^{\mathrm{b}}$, Lyndall Strazdins ${ }^{\mathrm{f}}$ \\ ${ }^{a}$ WZB Berlin Social Science Center, Reichpietschufer 50, 10785 Berlin, Germany \\ b Telethon KIDS Institute, The University of Western Australia, PO Box 855, West Perth, Western Australia 6872, Australia \\ ${ }^{c}$ Center for Population Health Research, Curtin University, GPO Box U1987, Perth, Western Australia, Australia \\ ' School of Nursing, Midwifery and Paramedicine, Curtin University, GPO Box U1987, Perth, Western Australia, Australia \\ e Menzies Institute for Medical Research, University of Tasmania, Private Bag 23, Hobart, Tasmania 7000, Australia \\ ${ }^{\mathrm{f}}$ Center for Epidemiology and Population Health, Research School of Population Health, The Australian National University, 62 Mills Road, Canberra, ACT \\ 2601, Australia
}

\section{A R T I C L E I N F O}

\section{Article history:}

Received 24 November 2016

Received in revised form

11 May 2017

Accepted 23 May 2017

Available online 26 May 2017

\section{Keywords:}

Maternal work hours

Fathers' work hours

Child BMI

Overweight

Obesity

Family income

The Raine Study

\begin{abstract}
A B S T R A C T
Using longitudinal data from the Western Australia Pregnancy Cohort (Raine) Study and both randomeffects and fixed-effects models, this study examined the connection between maternal work hours and child overweight or obesity. Following children in two-parent families from early childhood to early adolescence, multivariate analyses revealed a non-linear and developmentally dynamic relationship. Among preschool children (ages 2 to 5), we found lower likelihood of child overweight and obesity when mothers worked $24 \mathrm{~h}$ or less per week, compared to when mothers worked 35 or more hours. This effect was stronger in low-to-medium income families. For older children (ages 8 to 14), compared to working $35-40 \mathrm{~h}$ a week, working shorter hours $(1-24,25-34)$ or longer hours (41 or more) was both associated with increases in child overweight and obesity. These non-linear effects were more pronounced in lowto-medium income families, particularly when fathers also worked long hours.
\end{abstract}

๑) 2017 Elsevier Ltd. All rights reserved.

\section{Introduction}

The co-occurrence of increasing child overweight and obesity (Lobstein et al., 2015) with rising maternal employment (Baxter, 2013; Bureau of Labor Statistics, 2010; Labour Statistics Division, 2007) in the last three to four decades worldwide has drawn much attention to their possible connections (Anderson et al., 2003; Brown et al., 2010; Datar et al., 2014; Gwozdz et al., 2013; Hawkins et al., 2009; Morrissey, 2013; Phipps et al., 2006; ZiolGuest et al., 2013). Even while mothers' employment has

\footnotetext{
* Corresponding author. WZB Berlin Social Science Center, Reichpietschufer 50, 10785 Berlin, Germany.

E-mail address: jianghong.li@wzb.eu (J. Li).

1 Present affiliation: Department of Sociology and Human Geography, University of Oslo, Postboks 1096 Blindern, 0317 Oslo, Norway.
}

increased, mothers still retain primary responsibility for household work including meal provision and preparation (Jabs et al., 2007).

Previous studies show a positive association between mothers' work hours and child body mass index (BMI), independent of family socioeconomic and demographic characteristics (Anderson, 2012; Anderson et al., 2003; Datar et al., 2014). Constraints on mothers' time allocation for child health promoting activities have been viewed as the primary pathway linking maternal employment to childhood BMI. Longer maternal work hours have been linked to less time spent on grocery shopping and meal preparation (Cawley and Liu, 2012), greater consumption of prepared food (Cawley and Liu, 2012) and low quality diet (Li et al., 2011), and longer TV viewing hours and internet use in children (Datar et al., 2014; Fertig et al., 2009; Hawkins et al., 2009). Research has also examined these factors as mechanisms linking maternal employment to child BMI (Anderson, 2012; Brown et al., 2010; Datar et al., 2014; Fertig 
et al., 2009; Speirs et al., 2014; Ziol-Guest et al., 2013). However, most studies found only a weak mediating effect of these factors on child BMI (Datar et al., 2014; Fertig et al., 2009; Ziol-Guest et al., 2013). In one study no such evidence was found (Anderson, 2012). More recent research showed that it was children's night time sleep duration, but not TV time, diet quality or mealtime routines, which mediated the association between maternal work hours and child BMI (Speirs et al., 2014).

Several limitations are notable among previous studies on this topic. First, most previous research on maternal work hours and child BMI assumes that this connection is linear and similar for all developmental ages: the more hours mothers work, the less time they have and consequently the risk for child overweight increases. These studies analyse maternal work hours as a continuous variable. This approach implies a dose-response relationship, whereby any maternal employment is harmful to children's health. It neglects the possibility that mothers' employment may confer health benefits to the family. While some studies analysed maternal work hours as a categorical variable, only crude categories were examined, such as not employed, part-time and full-time (Bishop, 2011; Brown et al., 2010; Gwozdz et al., 2013), thus masking considerable variations within part-time and full-time work.

Second, although several studies have included children at different developmental stages (Anderson, 2012; Brown et al., 2010; Datar et al., 2014; Gwozdz et al., 2013; Morrissey, 2013), few have considered changes in the impact of maternal work on child BMI from early to middle childhood and adolescence (Morrissey, 2013; von Hinke Kessler Scholder, 2008). In Australia, where our study is based, the majority of mothers stay at home or work part-time during the preschool years (birth to age 5). When children commence schooling at age 6 family care time changes and mothers adjust their employment accordingly. A greater proportion of mothers return to work and many increase their work hours, suggesting that the relationship between mothers' work hours and child BMI may also change. Before children start school, when care needs are high, the threshold for "manageable" work hours is likely to be lower than when children begin schooling (9am to $3 \mathrm{pm}$ in Australia). However, mothers of school-aged children can still face significant time demands, as help with homework and extra curriculum activities (academic, music and sport) increase. Thus, long work hours or work hours above a threshold can be stressful and in turn have a negative impact on child health.

Third, with only a few exceptions (Anderson, 2012; Anderson et al., 2003; Morrissey, 2013), previous studies in this field are mainly based on cross-sectional data (Brown et al., 2010; Datar et al., 2014; Phipps et al., 2006; Speirs et al., 2014; Ziol-Guest et al., 2013). This has limited researchers' ability to address the issue of causality and consider how the work-hour -child BMI relationship changes as children develop and mothers alter their employment patterns.

\section{Theoretical considerations}

We propose that mothers' employment brings to families both gains and losses. The losses largely center on mothers' time, and the gains come from other resources, both material and psychological. Thus, rather than work hours creating a linear risk for child overweight, there may be tipping points in the maternal work hourchild weight relationship. Working at least some hours may be beneficial, depending on child developmental needs, and other family resources (e.g., income). We base our argument on the conceptual resource framework developed by Brooks-Gunn and colleagues (Brooks-Gunn et al., 1995) and the concept of work-tofamily fit (Tuttle and Garr, 2012) whereby work entails both conflicts and enrichment for families (Greenhaus and Beutell, 1985;
Greenhaus and Powell, 2006).

Based on multidisciplinary perspectives (economics, sociology, social demography, developmental and clinical psychology, and pediatrics), the conceptual resource framework identifies intrafamilial and extra-familial resources. Four categories of intrafamilial resources are considered to be critical for optimal child development and they include income, time, human and psychological capital. The psychological capital comprises parents' mental health, the quality of marital relationships or partnership, the psychological importance to them of education and work, and beliefs about the parental role in child rearing. Extra-familial resources include childcare settings, schools, peer groups, community, and wider social contexts (Kendall and Li, 2005). By engaging with these community resources appropriately parents can build social capital, another important resource for optimal child development. Parents face choices about allocating different domains of limited resources (Brooks-Gunn et al., 1995). When mothers participate in the labor market, they gain income, an important familial resource that enables the family to purchase healthy food (e.g., fresh produce and high quality low fat meat or fish), but at the same time other domains of familial resources may diminish, such as time available for meal preparation or engaging children with physical activity, and psychological capital, such as stress and fatigue associated with juggling between work and family. It is arguable that an optimal mixture of adequate resources is important for healthy child development.

The concept of work-to-family fit refers to the interface between work-family conflict (Greenhaus and Beutell, 1985) and workfamily enrichment (Greenhaus and Powell, 2006; Marshall and Barnett, 1993). Work-family conflict occurs when workers perceive or experience incompatibility between work and family domains, due to time, energy, and behavioural constraints and a psychological carryover of strain from work to family (Greenhaus and Beutell, 1985) or vice versa. From the work-family enrichment perspective however, employees gain instrumental resources from their work role, including interpersonal skills, coping skills, social capital, material goods, and psychological resources (job satisfaction, self-esteem, recognition, and optimism). These resources improve work performance and can benefit family functioning (e.g., parenting role) (Greenhaus and Powell, 2006; McNall et al., 2009). Much of the existing research on maternal employment and child outcomes is implicitly motivated by a conflict perspective and fails to consider that maternal employment can also confer benefits for working mothers and their children. These gains may be evident when mothers' work hours are not excessively long, and time pressures can be offset. The conflict may occur when mothers' capacities are exceeded, and other resources do not compensate for time constraints.

Research on workers' mental health suggests that there are thresholds whereby working at least some hours is good for health (Dooley and Prause, 2004), but only up to a point (Kleiner and Pavalko, 2010). Two cross-sectional studies found that young children (ages 4-7) whose mothers worked part-time watched less TV and were less likely to be overweight than children whose mothers who were not employed or worked 35 or more hours (Brown et al., 2010). Among 15-19 year olds both maternal part-time and fulltime employment was associated with decreases in overweight, compared to being not employed (Bishop, 2011). This literature suggests that there may be gains from maternal employment that counter balance constraints for family time, depending on the number of hours mothers work, and the developmental needs of children.

Motivated by the concept of work-family enrichment and workfamily conflict, we examined the non-linear effect of maternal work hours on child overweight and obesity and the dynamic of this 
effect across the child development period from early childhood (ages 2 to 5) to middle childhood and early adolescence (ages 8 to 14). The study was based on longitudinal data from the Western Australian Pregnancy Cohort (Raine) Study. The Raine Study collected repeated measures of maternal weekly work hours, child BMI and important time-varying and time-invarying covariates. We estimated both random-effects and fixed-effects models, considered multiple categories of mothers work hours, and controlled for a wide range of socioeconomic and health characteristics of the mother and child. Our study addresses four research questions: (1) Is there a threshold of maternal work hours for child BMI? (2) Does the relationship change during child development periods? (3) Does this relationship vary by income? (4) Does it differ by fathers' work hours? Previous studies show that as a covariate, fathers' work has no impact on child BMI. Most fathers work full time and there is far less variation in their work hours in general and fewer changes in work hours across the child developmental periods, compared to mothers' work hours. This is a methodological issue that reflects gender inequality. Two studies showed an interaction between maternal work hours and paternal work hours (Morrissey, 2013; Greve, 2011). While mothers are mainly responsible for meal planning and provision, fathers can assist in this task by doing other household work and attending children's needs for sport, music and social activities. However, when fathers work long hours, their capacity for supporting mothers in meeting children's nutritional needs is limited.

\section{Methods}

\subsection{Data}

The Raine Study is a prospective cohort study of 2868 live births. Between 1989 and 19912900 pregnant women were recruited at 18 weeks gestation through the State maternity hospital and private birth clinics nearby in Perth, Western Australia. Demographic, socioeconomic and health behavior data were collected through questionnaires answered by the primary carer and older children. Clinical, physical, and psychological assessments of the children were undertaken by clinicians and nurses at 34 weeks gestation, birth, ages 1, 2, 3, 5, 8, 10,14, 17, 20, and 22. Between ages 2 and 14 examined in our study, the average participation rate is $81.1 \%$ of the original cohort at birth $(n=2868)$. The Raine Cohort did not differ from the child and adolescent population of Western Australia on health and most of socio-demographic characteristics. Neither did the participants differ from drop-outs at each follow-up regarding premature birth, birthweight, and birth complications. These factors are important for child overweight and obesity (see supplementary Note 1 for more details). The Raine Study protocol was approved by the Human Research Ethics Committees at King Edward Memorial Hospital and Princess Margaret Hospital for Children in Perth.

The present study focused on preschool children (ages 2 to 5) and school-aged children (ages 8 to 14), and both-parent families as previous research suggests that it is important to consider fathers' work hours (Greve, 2011; Morrissey, 2013). The analysis included all follow-ups at which two parents were at present, but it was not a requirement that the child must live in a two-parent families at all follow-ups.

The missing cases due to item-nonresponse within follow-ups ranged $1 \%$ to $11.5 \%$ for ages $8-14$, and up to $19 \%$ for ages $2-5$. We used the multiple imputation procedure to impute the missing data. The imputation yielded two effective samples: for ages 2-5 $\mathrm{N}=1936$ children and 3396 observations; for ages 8-14 $\mathrm{N}=1801$ children and 4013 observations. All models were based on the imputed data (more details in supplementary Note 2).

\subsection{Dependent variable}

Children's body weight and height were measured by study nurses using calibrated scales and stadiometer at each follow-up. The BMI was calculated with adjustment for child age and gender and was re-coded into 4 categories - underweight, normal, overweight, and obese (Cole et al., 2000). A binary variable whether or not a child was overweight and obese versus normal weight was preferred over a continuous measure of BMI as we were mainly interested in the qualitative change from having a normal BMI score to being overweight and obese. Observations (child-follow-ups) which were classified as "underweight" were excluded from the analysis (2.4\% for ages $2-5$ and $0.9 \%$ for ages $8-14$ ). Preliminary analysis has shown that the results did not differ whether or not the analysis included the underweight observations.

\subsection{Key independent variables}

Guided by our theoretical arguments, we analysed mothers' work hours as a categorical rather than a continuous variable. Based on various preliminary analysis and the national average weekly work hours for women during the relevant time period, we coded mothers' weekly work hours as five categories for ages 8-14: not employed, 1-24 h, 25-34 h, 35-40 h (reference), and 41 or more hours (more details in supplementary Note 3 ). We used a similar approach to define four different work hour categories for ages 2 to 5: not employed, 1-24 h (reference), 25-34 h, and 35 or more hours based on a lower prevalence of maternal employment in early childhood. The likelihood of being overweight and obese was the lowest when mothers worked between 1 and $24 \mathrm{~h}$ per week before children entered school and 35-40 h for school-aged children (Table 1).

\subsection{Control variables}

In all analyses we adjusted for family income, maternal education and occupation, and fathers' work hours. Demographic covariates and relevant health variables were also controlled for to minimise potential selection bias associated with them, including maternal age at the child's birth, maternal race, maternal mental health, maternal BMI, maternal smoking, child gender, child birth weight, and the number of siblings in the family.

\subsubsection{Time-variant variables}

Fathers' work hours were collected from age 5 onwards and were coded in 5 categories (not employed, working 1-34 h, $35-44 \mathrm{~h}, 45-54 \mathrm{~h}$ and 55 or more hours per week). Before the child reached age 5 only fathers' employment status was recorded. Information on the gross annual household income was collected as an ordinal variable at every follow-up but the range differed across follow-ups. A new variable was created that was reasonably comparable across follow-ups: top 30 per cent, middle 30 per cent, and bottom 40 per cent for ages 2 to 3; the top 40 per cent, middle 30 per cent, and the bottom 30 per cent for age 5; for ages 8 to 14, low income (bottom 10\%), lower middle income (next 30\%), higher middle income (next 25\%), and high income (top 35\%). A binary variable, whether or not a mother was treated for mental health problem ( $1=$ yes, $0=$ no), was controlled for ages 8 to 14 , but this information was not collected at ages 2 to 5 . The number of siblings was controlled for as a categorical variable $(0,1$, and 2 or more siblings). Dummy variables for each follow-up were included in the analysis to adjust for the natural increase in mothers' work hours and child overweight over time, or possible changes in the social and economic environment across the follow-ups. 
Table 1

Mother's work hours by child overweight status.

\begin{tabular}{|c|c|c|c|c|c|c|c|}
\hline \multicolumn{4}{|l|}{ Ages $2-5$} & \multicolumn{4}{|l|}{ Ages $8-14$} \\
\hline & $\begin{array}{l}\text { Normal } \\
\mathrm{N}(\%)\end{array}$ & $\begin{array}{l}\text { Overweight/Obese } \\
\mathrm{N}(\%)\end{array}$ & Total & & $\begin{array}{l}\text { Normal } \\
\mathrm{N}(\%)\end{array}$ & $\begin{array}{l}\text { Overweight/Obese } \\
\mathrm{N}(\%)\end{array}$ & Total \\
\hline $\begin{array}{l}\text { Mother's work hours: } \\
\text { Not employed }\end{array}$ & $\begin{array}{l}801 \\
(87.5)\end{array}$ & $\begin{array}{l}115 \\
(12.5)\end{array}$ & 916 & $\begin{array}{l}\text { Mother's work hours: } \\
\text { Not employed }\end{array}$ & $\begin{array}{l}846 \\
(79.5)\end{array}$ & $\begin{array}{l}218 \\
(20.5)\end{array}$ & 1,3064 \\
\hline $1-24$ & $\begin{array}{l}676 \\
(89.2)\end{array}$ & $\begin{array}{l}82 \\
(10.8)\end{array}$ & 758 & $1-24$ & $\begin{array}{l}910 \\
(78.7)\end{array}$ & $\begin{array}{l}247 \\
(21.3)\end{array}$ & 1157 \\
\hline $25-34$ & $\begin{array}{l}140 \\
(84.3)\end{array}$ & $\begin{array}{l}26 \\
(15.7)\end{array}$ & 166 & $25-34$ & $\begin{array}{l}380 \\
(76.2)\end{array}$ & $\begin{array}{l}119 \\
(23.8)\end{array}$ & 499 \\
\hline$\geq 35$ & $\begin{array}{l}218 \\
(82.9)\end{array}$ & $\begin{array}{l}45 \\
(17.1)\end{array}$ & 263 & $35-40$ & $\begin{array}{l}330 \\
(80.5)\end{array}$ & $\begin{array}{l}80 \\
(19.5)\end{array}$ & 410 \\
\hline Total & $\begin{array}{l}1835 \\
(87.3)\end{array}$ & $\begin{array}{l}268 \\
(12.7)\end{array}$ & 2103 & $\geq 41$ & $\begin{array}{l}115 \\
(68.0)\end{array}$ & $\begin{array}{l}54 \\
(32.0)\end{array}$ & 169 \\
\hline & & & & Total & $\begin{array}{l}2581 \\
(78.2)\end{array}$ & $\begin{array}{l}718 \\
(21.8)\end{array}$ & 3299 \\
\hline
\end{tabular}

Categories used as reference categories in regression models are bolded.

\subsubsection{Time-invariant variables}

Child gender was included in the analysis as a dichotomy: $0=$ girl and $1=$ boy. Maternal education was recoded into six categories: no secondary education, trade certificate, professional registration, college diploma, university degree, other type (more details in Note 4). Maternal occupation was included as a proxy for job characteristics with six categories: not employed, managers and professionals, para-professionals and trades workers, clerical workers, sales workers, operators and laborers. Maternal age (14-24, 25-29, 30-34 and 34 years or over) at the child's birth and maternal ethnicity was controlled for (Caucasian vs. other). Maternal BMI ("normal", "overweight" and "obese") and maternal smoking (not smoking, 1-10 cigarettes daily, 11 or more cigarettes daily) during pregnancy were controlled for genetic predisposition and a proxy for dietary habits and lifestyles in the home. A dummy indicating if the child attended child care was included in the analyses of preschool-aged children.

\subsection{Analytical methods}

We analysed both random-effects (RE) and fixed-effects (FE) logit models because each of these models offers comparative advantages and disadvantages (Petersen, 2004, p. 342). The FE model addresses bias due to unobserved heterogeneity because it takes into account only within-individual variation in the dependent and independent variables. However, as the FE mode excludes all observations without temporal variation in the dependent or independent variables, its estimation is less efficient than the RE model and the standard errors are larger due to the reduced sample size. The RE model takes into account both within and between individual variation, and it makes use of all observations including those which are measured at only one time point. Consequently, the RE model is considered more efficient than the FE model, but its estimation is unbiased only if the omitted time-constant variables captured in the error term are uncorrelated with the independent variables. The Hausman test is conventionally used to determine whether the coefficients of the RE model are consistent (null hypothesis), given that the FE model coefficients are consistent, or whether only the FE model is consistent (alternative hypothesis) (Greene, 2011; Hausman, 1978). If the null hypothesis is not rejected, the RE model is preferred due to its higher efficiency.

We began the analysis with RE and FE models controlling for the wide range of covariates, separately for preschool children and older children. In the second step, we stratified the analysis by family income. Previous research suggests that the impact of maternal work hours on child BMI differs by family socioeconomic status, with a stronger effect in high SES families than in families with low to medium SES (Anderson et al., 2003; Datar et al., 2014). We estimated two sets of models: one for low income and the other for high income families for both preschool and school children. Using a similar approach as Datar et al. (2014), the sample was stratified into the bottom half (50\%) as low income and the top half (50\%) as the high income group for ages 8 to 14 , and the bottom 60 per cent as low income and the top 40 per cent as high income for ages 2 and 3. For age 5 due to the different income categories collected, the reasonable division was roughly the bottom 50 per cent vs top 50 per cent. The analyses were conducted using Stata 14.1 MP-Parallel Edition.

\section{Results}

At age 2, 5 per cent of the cohort was overweight and obese but this increased to11 per cent at age 3 and 15 per cent at age 5 (supplementary Table 1A). The prevalence of overweight and obesity continued to rise after children commenced schooling: 18 per cent at age 8,25 per cent at age 10 and it declined somewhat to 24 per cent at age 14. Maternal work hours also increased substantially between ages 2 and 14. At age 2, 68 per cent of the mothers stayed at home, while at age 14 this decreased to 22.6 per cent. At age 2 , only 7 per cent of the mothers worked $35 \mathrm{~h}$ or more per week, while at age 14 this increased to 25.6 per cent. In contrast, relatively few changes occurred for fathers. Between ages 2 to $5,12.5$ per cent of the fathers were not employed, and this decreased to 8 per cent when their child reached ages 8 to 14 . Other socioeconomic and demographic characteristics of the cohort are shown in the supplementary Tables $1 \mathrm{~A}$ and Table $1 \mathrm{~B}$.

\subsection{Multivariate results}

\subsubsection{Preschool children (ages 2 to 5)}

To avoid common misinterpretation of odds ratios as pointed out by some (Holcomb et al., 2001; Davies, 1998), we presented the parameter estimates as the log of odds (logit) rather than odds ratios (Table 2): for categorical variables a negative logit corresponds to an odds ratio below 1 and vice versa. To facilitate the interpretation of logits, we presented marginal effects as recommended by experts (Fullerton, 2016). Marginal effects indicate the change in the predicted probability for the respective category compared to the reference category.

Compared to working $1-24 \mathrm{~h}$, if mothers worked 35 or more hours per week there was an increased risk for child overweight or obesity. Being not employed or working 25-34 h was not 
Table 2

Mother's work hours and child overweight and obesity: Ages 2 to 5 . $^{\text {a }}$

\begin{tabular}{|c|c|c|}
\hline & \multicolumn{2}{|c|}{ Random-effects } \\
\hline & $\begin{array}{l}\text { Log odds } \\
\text { (SE) }\end{array}$ & $\begin{array}{l}\text { Marginal effects } \\
\text { (SE) }\end{array}$ \\
\hline $\begin{array}{l}\text { Mother's work hours } \\
\text { Not employed } \\
1-24 \text { (ref.) }\end{array}$ & $\begin{array}{l}-0.14 \\
(0.60)\end{array}$ & $\begin{array}{l}0.00 \\
(0.01)\end{array}$ \\
\hline $25-34$ & $\begin{array}{l}0.48 \\
(0.43)\end{array}$ & $\begin{array}{l}0.02 \\
(0.02)\end{array}$ \\
\hline$\geq 35$ & $\begin{array}{l}\mathbf{0 . 8 6}^{*} \\
(0.37)\end{array}$ & $\begin{array}{l}0.02 \\
(0.01)\end{array}$ \\
\hline Observations & 3396 & 2105 \\
\hline Groups & 1936 & 1482 \\
\hline
\end{tabular}

$+: \mathrm{p}<0.10 ;^{*}: \mathrm{p}<0.05 ;^{* *}: \mathrm{p}<0.01$.

a Covariates included fathers' employment, family income, number of siblings, maternal education, occupation, age, ethnicity, BMI before pregnancy, smoking, child gender and birth weight and follow up. The parameter estimates are in Supplementary Tables.

b Marginal effects: the difference between the predicted probability for respective categories and that for the reference category.

significantly different from the reference group. Working $35 \mathrm{~h}$ or more per week was associated with an increase in the probability of overweight or obesity by 2 percentage points. The prevalence of overweight and obesity for $1-24 \mathrm{~h}$ (reference) is 11 per cent. A 2 per cent increase due to the change to 35 or more hours would increase the prevalence to 13 per cent, an 18 per cent increase which is nontrivial. At a population level, this represents a further $2 / 100$ children who move into overweight or obesity, highly consequential for lifetime risk and burden of disease.

In the FE model (supplementary S-Table 2), maternal work hours exhibited a similar non-linear relationship with the dependent variable, although this association did not reach significance. This was likely due to lack of power given the substantial reduction in observations ( 358 child-follow-ups) and groups (165 children), compared to the RE model (3396 observations and 1936 groups). Based on the Hausman test $(\mathrm{p}=0.99)$, the estimates from both RE and RE models were consistent, therefore the RE model was preferred.

Notably a number of covariates were strongly associated with the dependent variable (supplementary S-Table 2). Maternal overweight and obesity before pregnancy had a strong positive effect on child overweight and obesity. Similarly, heavy maternal smoking in pregnancy ( 11 or more cigarettes per day) was strongly associated with child overweight and obesity. Boys were less likely to be overweight and obese than girls and child birth weight was positively associated with child overweight and obesity.

Table 3 shows the adjusted results from the RE, stratified by family income. In the low income group, compared to working 1-24 h weekly, working 35 or more hours was strongly associated with a higher risk for child overweight or obesity (beta $=1.61$, $\mathrm{p}<0.01$ ) and an increase in the probability of overweight or obesity by 5 percentage points. In the high income group there was no significant association between maternal work hours and child overweight, although there was a non-linear pattern (S-Table 3). Based on the Hausman test $(p=0.94)$, the estimates from the random-effects model were preferred for both income groups.

\subsubsection{Late childhood and early adolescence (ages 8 to 14)}

Multivariate results for ages 8 to 14 are shown in Table 4. They reveal a similar non-linear relationship between mothers' work hours and child BMI, but the work hour threshold was higher. Compared to mothers' working 35-40 h per week, 41 or more hours were significantly and strongly associated with the likelihood of child overweight and obesity in both models. In the RE model, work hours less than the reference category (35-40 h) were associated with greater likelihood of child overweight and obesity: $1-24 \mathrm{~h}(\mathrm{p}<0.05)$ and $24-34 \mathrm{~h}(\mathrm{p}<0.10)$. In the FE model the effect of these two work hour categories below the reference did not reach statistical significance, although the non-linear relationship remained. However, the effect of working 41 or more hours strengthened in the FE model. Based on the Hausman test ( $p \leq 0.001$ ), the estimates from the RE model were not consistent and hence the FE model was preferred. The marginal effects in the FE model showed that being not employed or working below the threshold (1-24 or $25-34 \mathrm{~h}$ ) were associated with an increase in the probability of child overweight or obesity by 7, 9 and 8 percentage points, respectively, compared to the reference. Working 41 or more hours was associated with a drastic increase by 20 percentage points.

In contrast, fathers' work hours alone had no effect on child overweight and obesity, but a number of covariates were significantly associated with child BMI (supplementary S-Table 3). Maternal overweight and obesity before pregnancy, maternal smoking during pregnancy and child birth weight were all positively and strongly associated with child overweight or obesity. Children with three or more siblings were less likely to be overweight and obese.

Table 5 shows the multivariate results stratified by family income, with all covariates adjusted for. The non-linear relationship between maternal work hours and child overweight or obesity was apparent in the low income households in the RE. In these families, the lowest risk for child overweight or obesity was when mothers worked 35-40 h weekly: the risk was higher for work hours below or above this threshold. Being not employed or working 1-24 h or 25-34 h were associated with an increased probability of child overweight or obesity by $4-5$ percentage points; working hours above the threshold was associated with an increase by 12 percentage points. Among high income families, we found only one consistent albeit weaker association $(p<0.10)$ : compared with working 35-40 h per week, working 41 or more hours was associated with higher likelihood of overweight and obesity. According to the Hausman test ( $p=0.95$ ), the estimates from the RE model were consistent with those from the FE model (supplementary STable 5) and hence the RE estimates were preferred.

Thus far our results show that fathers' work hours on their own have no significant effect on child BMI. In further analysis (supplementary S-Table 6), we stratified the analysis by fathers' long work hours ( $<45$ vs $\geq 45$ h per week) for school-aged children. Based on the RE models, the non-linear effects of mothers' work hours on child weight remained largely unchanged when fathers worked below $45 \mathrm{~h}$ in the low income families. However, when fathers worked 45 or more hours, the detrimental effect of long maternal work hours (41 or more) and short hours (1-24) strengthened. For the high income families there was no difference regardless of whether or not fathers worked 45 or more hours (available on request). The numbers of observations in the stratified models were too small for FE estimates to be reliable.

\subsubsection{Sensitivity tests}

Maternal physical illness might confound the non-linear effect of maternal work hours on child bodyweight. Data for this variable was only collected at follow-ups 10 and 14 . We tested for two health indicators: (1) whether mother was limited in daily activities because of health problem; (2) an ordinal variable indicating any long term health problems (poor, fair, good, very good, and excellent). Both illness indicators were significantly associated with child overweight or obesity, but they did not modify the non-linear effect of maternal work hours on the outcome variable (results 
Table 3

Mother's work hours and child overweight and obesity: Ages 2 to 5 by income.

\begin{tabular}{|c|c|c|c|c|}
\hline & \multicolumn{2}{|c|}{ Low income } & \multicolumn{2}{|c|}{ High income } \\
\hline & \multicolumn{2}{|c|}{ Random-effects } & \multicolumn{2}{|c|}{ Random-effects } \\
\hline & $\begin{array}{l}\text { Log Odds } \\
(\mathrm{SE})\end{array}$ & $\begin{array}{l}\text { Marginal effects } \\
(\mathrm{SE})\end{array}$ & $\begin{array}{l}\text { Log odds } \\
(\mathrm{SE})\end{array}$ & $\begin{array}{l}\text { Marginal effects } \\
(\mathrm{SE})\end{array}$ \\
\hline \multicolumn{5}{|c|}{ Mother's work hours } \\
\hline Not employed & $\begin{array}{l}-0.03 \\
(0.86)\end{array}$ & $\begin{array}{l}0.02 \\
(0.01)\end{array}$ & $\begin{array}{l}0.23 \\
(0.92)\end{array}$ & $\begin{array}{l}0.00 \\
(0.01)\end{array}$ \\
\hline \multicolumn{5}{|l|}{$1-24$ (ref.) } \\
\hline $25-34$ & $\begin{array}{l}0.47 \\
(0.68)\end{array}$ & $\begin{array}{l}0.02 \\
(0.03)\end{array}$ & $\begin{array}{l}0.60 \\
(0.62)\end{array}$ & $\begin{array}{l}0.01 \\
(0.02)\end{array}$ \\
\hline$\geq 35$ & $\begin{array}{l}1^{62} 2^{* *} \\
(0.59)\end{array}$ & $\begin{array}{l}0.05 \\
(0.03)\end{array}$ & $\begin{array}{l}0.39 \\
(0.54)\end{array}$ & $\begin{array}{l}0.01 \\
(0.01)\end{array}$ \\
\hline Observations & 1854 & 1050 & 1391 & 1058 \\
\hline Groups & 1266 & 842 & 948 & 798 \\
\hline
\end{tabular}

$+: \mathrm{p}<0.10 ;{ }^{*}: \mathrm{p}<0.05 ;^{* *}: \mathrm{p}<0.01$.

a Adjusted as for same covariates as in Table 2. The parameter estimates are in Supplementary Tables.

Table 4

Mother's work hours and child overweight and obesity: Ages 8 to 14 . $^{\mathrm{a} b}$

\begin{tabular}{|c|c|c|c|c|}
\hline & \multicolumn{2}{|c|}{ Random-effects } & \multicolumn{2}{|c|}{ Fixed-effects } \\
\hline & $\begin{array}{l}\text { Log odds } \\
\text { (SE) }\end{array}$ & $\begin{array}{l}\text { Marginal effects } \\
(\mathrm{SE})\end{array}$ & $\begin{array}{l}\text { Log odds } \\
\text { (SE) }\end{array}$ & $\begin{array}{l}\text { Marginal effects } \\
\text { (SE) }\end{array}$ \\
\hline Mother's work hours & -0.23 & 0.01 & 0.30 & 0.07 \\
\hline Not employed & $(0.70)$ & $(0.01)$ & $(0.47)$ & $(0.07)$ \\
\hline $1-24$ & $\begin{array}{l}\text { 0.67* } \\
(0.32)\end{array}$ & $\begin{array}{l}\text { 0.02* } \\
(0.01)\end{array}$ & $\begin{array}{l}0.54 \\
(0.42)\end{array}$ & $\begin{array}{l}0.09+ \\
(0.06)\end{array}$ \\
\hline $25-34$ & $\begin{array}{l}0.62+ \\
(0.34)\end{array}$ & $\begin{array}{l}0.02+ \\
(0.01)\end{array}$ & $\begin{array}{l}0.49 \\
(0.41)\end{array}$ & $\begin{array}{l}0.08 \\
(0.06)\end{array}$ \\
\hline $\begin{array}{l}35-40 \text { (ref.) } \\
\geq 41\end{array}$ & $\begin{array}{l}1^{.61 * *} \\
(0.48)\end{array}$ & $\begin{array}{l}\mathbf{0 . 0 7} \\
(0.03)\end{array}$ & $\begin{array}{l}\mathbf{2 . 0 7}{ }^{* *} \\
(0.75)\end{array}$ & $\begin{array}{l}\text { 0.20** } \\
(0.06)\end{array}$ \\
\hline Observations & 4013 & 3464 & 621 & 568 \\
\hline Groups & 1801 & 1594 & 228 & 210 \\
\hline
\end{tabular}

$+: \mathrm{p}<0.10 ;^{*}: \mathrm{p}<0.05 ;{ }^{* *}: \mathrm{p}<0.01$.

a Adjusted for same covariates as in Table 2, except child care and paternal employment (replaced by work hours). The parameter estimates are in Supplementary Tables.

b Hausman test suggests the fixed-effects model is preferred.

Table 5

Mother's work hours and child overweight and obesity: Ages 8 to 14 by income. ${ }^{a}$

\begin{tabular}{|c|c|c|c|c|}
\hline & \multicolumn{2}{|c|}{ Low income } & \multicolumn{2}{|c|}{ High income } \\
\hline & \multicolumn{2}{|c|}{ Random-effects } & \multicolumn{2}{|c|}{ Random-effects } \\
\hline & $\begin{array}{l}\text { Log odds } \\
(\mathrm{SE})\end{array}$ & $\begin{array}{l}\text { Marginal effects } \\
\text { (SE) }\end{array}$ & $\begin{array}{l}\text { Log odds } \\
(\mathrm{SE})\end{array}$ & $\begin{array}{l}\text { Marginal effects } \\
\text { (SE) }\end{array}$ \\
\hline Mother's work hours & 0.94 & $0.04^{*}$ & -1.91 & -0.01 \\
\hline Not employed & $(0.97)$ & $(0.02)$ & $(1.26)$ & $(0.01)$ \\
\hline \multirow[t]{2}{*}{$1-24$} & $1.41^{*}$ & 0.04* & 0.18 & 0.01 \\
\hline & $(0.57)$ & $(0.02)$ & $(0.42)$ & $(0.01)$ \\
\hline \multirow[t]{2}{*}{$25-34$} & $1.44^{*}$ & $0.05^{*}$ & 0.27 & 0.01 \\
\hline & $(0.61)$ & $(0.02)$ & $(0.43)$ & $(0.01)$ \\
\hline \multicolumn{5}{|l|}{$35-40$ (ref.) } \\
\hline$\geq 41$ & $\begin{array}{l}\text { 2.83** } \\
(0.86)\end{array}$ & $\begin{array}{l}\mathbf{0 . 1 2}^{*} \\
(0.06)\end{array}$ & $\begin{array}{l}1.03+ \\
(0.60)\end{array}$ & $\begin{array}{l}0.05 \\
(0.03)\end{array}$ \\
\hline Observations & 2020 & 1745 & 1993 & 1719 \\
\hline Groups & 1125 & 992 & 1027 & 901 \\
\hline
\end{tabular}

$+: \mathrm{p}<0.10 ;{ }^{*} \mathrm{p}<0.05 ;{ }^{* *} \mathrm{p}<0.01$.

a Adjusted for same covariates as in Table 4. The parameter estimates are in Supplementary Tables.

available on request).

We have also tested for the interaction between maternal occupation and work hours by stratifying the analysis by occupation (professional/manager vs other occupations). We observed the same non-linear association between maternal work hours and child overweight or obesity in both age groups. Long maternal work hours ( $\geq 35 \mathrm{~h}$ for ages $2-5$, and $\geq 41 \mathrm{~h}$ for ages $8-14$ ) were associated with higher likelihood of child overweight and obesity in both occupational groups (results available on request). We stratified the analysis by maternal education (tertiary versus no tertiary 
education) and found a similar non-linear relationship for both age groups to that based on the stratification by income (supplementary S-Tables 7 and 8).

\section{Discussions}

This study has demonstrated three key new findings. First, the study shows a non-linear relationship between mothers' work hours and child overweight status. For school-aged children 35-40 h per week appeared to be a beneficial threshold whereby hours that fell below or above were associated with greater likelihood of child overweight and obesity. This finding questions assumptions that (any) maternal employment is a risk for child overweight and obesity. Second, the maternal work hour threshold is dynamic and appears to be calibrated to child developmental needs. In early childhood working 35 or more hours confers a risk for child health. When children reached ages 8 to 14 , the threshold was 35-40 h per week. This dynamic likely reflects changes in child care needs, family routines and demands as children enter school (see Note 6). Third, the strength of the association between maternal work hours and child body weight differed by family income. Among low-to-medium income families, especially in school-aged children, the non-linear effect of maternal work hours was pronounced. Being not employed at all and working fewer than 35-40 h a week were associated with higher likelihood of child overweight and obesity, as was working above them. In these families the extra income earned by mothers may be important for household finances and protective of child weight, but only up to a point, when time constraints can no longer be counterbalanced. Consistent with this interpretation, we observed no negative effects when mothers worked short hours or not at all in the high income families (presumably because income is less critical in these families). However, when mothers worked more than 40 h each week, the risk of child overweight and obesity also emerged in the high income families, although to a lesser degree.

These findings support our broad hypotheses that the effect of maternal work hours on child health is non-linear and dynamic. They are consistent with the three theoretical perspectives that motivated this study, namely the conceptual resource framework (Brooks-Gunn et al., 1995), work-family enrichment (Greenhaus and Powell, 2006) and work-family conflict (Greenhaus and Beutell, 1985) theories. Although we were unable to directly test the conceptual resource framework, the non-linear effect of maternal work hours on child overweight and obesity is in line with the core argument of the framework: there are diverse and sometime conflicting family resources for optimal child development, such as income, time, parental mental wellbeing and social capital, and it is the optimal mix of these resources, not the quantity of a single resource, that makes a difference.

Consistent with the work-family enrichment perspectives, our results show that when mothers work at least some hours there appears to be benefits for children's body weight. This suggests that having more time available in the home by not participating in the labour force does not necessarily enable mothers to carry out activities that promote healthy child body weight. It is plausible that time constraints imposed by employment are offset by other benefits, at least up to a certain point. This is reflected in the work hour thresholds our analyses have identified.

Very few other studies have modelled non-linear patterns of the association between maternal working hours and child BMI. Our findings are consistent with limited prior research conducted outside the US. Greve (2011) found that maternal full time work ( $37 \mathrm{~h}$ per week) was associated with a lower risk of child overweight, compared to working 27-36 h per week in Denmark. Gwozdz et al. (2013) found that it was full-time work hours that were associated with higher risk for child overweight and obesity in eight European countries. Brown et al. (2010) showed that mothers' part-time work hours was associated with less TV viewing and lower likelihood of overweight in 4-5 year old Australian children, compared to being not employed. These studies used cross-sectional data and crude categories of maternal hours. However, they suggest that the nature of the work hour-child BMI relationship may differ by social and economic context at the country level. Our findings extend this evidence and show that among school children from low income families, mothers' fulltime employment is beneficial for child body weight. This supports our argument that employment supplies countervailing and protective resources even while it constrains mothers' time. These may be income but also psychological resources (e.g., social support, a valued identity, self-efficacy and optimism) that are protective of child weight, especially in low-to-medium income families. These hypotheses should be tested in future research. Among low income families, we found higher likelihood of child overweight and obesity if mothers worked few or no hours, a finding that was not observed among high income families. The financial benefits of full-time maternal employment may enable a low income household to afford fresh produce and good quality meats (which are especially costly in Western Australia due to the scarcity of water resources and isolation of the city) and purchase household services (e.g., cleaning) so mothers can preserve their time for meal preparation. Through employment these mothers may also gain skills and knowledge for promoting healthy living in the home (Watts et al., 2016), and psychological resources that have been linked to better food environments and child diet quality (Jarman et al., 2014). It is plausible that the relative psychological and monetary gains of full time employment are greater for low-tomedium income mothers than for mothers from high income families. This would explain the weaker non-linear effect of maternal work hours on child body weight among the high income families in our study.

Our study lends support to the work-family conflict argument by showing that long maternal work hours (developmentally different) are detrimental to child body weight. We observed this effect in both young and older children. Taken together, our findings suggest that neither work-family conflict nor work-family enrichment alone is adequate for understanding the nature of the maternal work-child health relationship. Rather we need to see both sides of the "coin" and consider both advantages and disadvantages associated with maternal employment. This is important not only in the way we model maternal work hour-child wellbeing relationships but also in conceptualising mechanisms that underpin them. The non-linear nature of the influence of maternal work hours on child BMI, as our study has revealed, may offer an explanation for the inconclusive findings about mechanisms thought to link maternal work hours to increases in child BMI (Anderson, 2012; Brown et al., 2010; Datar et al., 2014; Fertig et al., 2009; Ziol-Guest et al., 2013). Gains from maternal employment may counter balance time constraints and stresses so that child TV viewing or eating take-away food may not necessarily increase with maternal work hours in a linear manner (Brown et al., 2010). The relationship between maternal work hours and child health requires more complex and nuanced models that take into account the resources that employment may deliver, the constraints it may impose, and the developmental needs of children as they grow.

\section{Strengths and limitations}

This study was based on rare longitudinal cohort data covering early and middle childhood and early adolescence. The findings are robust against the adjustment for a wide range of socio-economic, 
demographic and health covariates. They are also robust against different estimation methods (random- and fixed-effects modelling). However, the families we studied lived in Western Australia and maternal employment patterns and the social support system for working parents may differ between Australia and other developed countries. Thus, the generalizability of our findings is yet to be established.

We were unable to directly test the mechanisms (e.g., psychological attributes, self-efficacy, and work and life satisfaction) that may underpin the non-linear relationship between maternal work hours and child body weight, due to lack of data. Maternal selfefficacy was only collected at the child's age 3 in the Raine Study. It would be important for future research to investigate these mechanisms. Further research is warranted to examine a more adequate measure of job characteristics and its potential interaction with maternal work hours. The non-representative sampling and attrition (although relatively low) limit the generalizability of our results. Thus, it is important to replicate our study with larger, national cohort datasets. However, with the attrition adjusted, our findings could become even stronger because the prevalence of child overweight and obesity is higher among mothers with lower education and the nonlinear effect of maternal work hours is more pronounced in families with lower maternal education as we have found.

Despite of its limitations, the Raine Study remains a valuable dataset for answering our research questions because it provides rare longitudinal clinical assessments of child BMI over different child development stages, and clinical measures of important maternal health covariates.

\section{Conclusions}

Our study provides novel, longitudinal evidence with important implications for future theory, research, and policy in this field. Child overweight and obesity rates continue to increase especially among the very young (42 million young children are estimated to be overweight and obese globally in 2013) (WHO, 2014). We show that maternal employment up to a certain threshold seems beneficial for child health particularly in low-medium income families. Our findings call for further research that will examine the ways in which employment enables lower income mothers to promote child health or constrains their ability to do so and factors that hinder or limit their participation in the labour force. Long work hours in contrast is detrimental for children's weight and especially when fathers work long hours in low income families. Child health is not the sole responsibility of the mother. The gender inequality that hinders a greater share of fathers operates both within the home and in the labour market, where fathers are expected to work at minimum full-time and often long hours. In countries with strong employment protections and work time regulations, both women and men work shorter hours, and gender equality is maximised (Landivar, 2015). Our study indicates that, along with gender equality in work hours, children's overweight, and the future burden of non-communicable disease that it predicts, could also decrease.

\section{Acknowledgements}

We thank Raine Study participants and their families for ongoing participation in the study; the Raine Study Team for coordination of the study and for the collection of the data presented here; the National Health and Medical Research Council (NHMRC) of Australia for their contribution to funding for the Raine study over the last 25 years; and the Telethon Kids Institute for their long term support. We acknowledge the University of
Western Australia (UWA), the Raine Medical Research Foundation, the UWA Faculty of Medicine, Dentistry and Health Sciences, the Women's and Infant's Research Foundation (KEMH), Curtin University and Edith Cowan University for providing funding for the Core Management of the Raine Study. Lyndall Strazdins is supported by an Australian Research Council Future Fellowship FT110100686, and this paper was part of a visiting fellowship supported by WZB Berlin Social Science Center (Wissenschaftszentrum Berlin für Sozialforschung).

\section{Appendix A. Supplementary data}

Supplementary data related to this article can be found at http:// dx.doi.org/10.1016/j.socscimed.2017.05.046.

\section{References}

Anderson, P., 2012. Parental employment, family routines and childhood obesity. Econ. Hum. Biol. 10, 340-351. http://dx.doi.org/10.1016/j.ehb.2012.04.006.

Anderson, P., Butcher, K., Levine, P.B., 2003. Maternal employment and overweight children. J. Health Econ. 22, 477-504. http://dx.doi.org/10.1016/S0167-6296(03) 00022-5.

Baxter, J., 2013. Child Care Participation and Maternal Employment Trends in Australia. (Research Report No. 26). Australian Institute of Family Studies, Melbourne.

Bishop, James, 2011. The effect of maternal employment on youth overweight in Australia. Econ. Rec. 87 (Special Issue), 92-104. http://dx.doi.org/10.1111/j.14754932.2011.00747.x.

Brooks-Gunn, J., Brown, B., Duncan, G.J., Moore, K.A., 1995. Child development in the context of family and community resources: an agenda for national data collections. In: Integrating Federal Statistics on Children: Report of a Workshop. National Academies Press, Washington, DC, pp. 27-95.

Brown, J.E., Broom, D.H., Nicholson, J.M., Bittman, M., 2010. Do working mothers raise couch potato kids? Maternal employment and children's lifestyle behaviours and weight in early childhood. Soc. Sci. Med. 70, 1816-1824. http://dx.doi.org/ 10.1016/j.socscimed.2010.01.040.

Bureau of Labor Statistics, 2010. The Economics Daily: Labor Force Participation Rates Among Mothers. U.S. Department of Labor. http://www.bls.gov/opub/ted/ 2010/ted 20100507.htm (Accessed 30 June 2016).

Cawley, J., Liu, F., 2012. Maternal employment and childhood obesity: a search for mechanisms in time use data. Econ. Hum. Biol. 10, 352-364. http://dx.doi.org/ 10.1016/j.ehb.2012.04.009.

Cole, T.J., Bellizzi, M.C., Flegal, K.M., Dietz, W.H., 2000. Establishing a standard definition for child overweight and obesity worldwide: international survey. Brit. Med. J. 320, 1240-1243. http://dx.doi.org/10.1136/bmj.320.7244.1240.

Datar, A., Nicosia, N., Shier, V., 2014. Maternal work, children's diet, activity, and obesity. Soc. Sci. Med. 107, 196-204. http://dx.doi.org/10.1016/ j.socscimed.2013.12.022.

Davies, H.T.O., 1998. When can odds ratios mislead? Br. Meical J. 316, 989.

Dooley, D., Prause, J., 2004. The Social Costs of Underemployment: Inadequate Employment as Disguised Unemployment. Cambridge University Press, Cambridge.

Fertig, A., Glomm, G., Tchernis, R., 2009. The connection between maternal employment and childhood obesity: inspecting the mechanisms. Rev. Econ. Househ. 7, 227-255. http://dx.doi.org/10.1007/s11150-009-9052-y.

Fullerton, A., 2016. Ordered Regression Models Workshop (June 29, 2016). Berlin.

Greene, W.H., 2011. Econometric Analysis, seventh ed. (Prentice Hall, New York).

Greenhaus, J.H., Beutell, N.J., 1985. Sources of conflict between work and family roles. Acad. Manage. Rev. 10, 76-88. http://dx.doi.org/10.5465/ AMR.1985.4277352.

Greenhaus, J.H., Powell, G.N., 2006. When work and family are allies: a theory of work-family enrichment. Acad. Manage. Rev. 31, 72-92. http://dx.doi.org/ 10.5465/AMR.2006.19379625.

Greve, J., 2011. New Results on the effects of maternal work hours on children's overweight status: does the quality of child care matter? Labour Econ. 18, 579-590. http://dx.doi.org/10.1016/j.labeco.2011.03.003.

Gwozdz, W., Sousa-Poza, A., Reisch, L.A., Ahrens, W., Eiben, G., FernandézAlvira, J.M., , et al.Veidebaum, T., 2013. Maternal employment and childhood obesity - a European perspective. J. Health Econ. 32, 728-742. http:// dx.doi.org/10.1016/j.jhealeco.2013.04.003.

Hausman, J.A., 1978. Specification tests in econometrics. Econometrica 46, 1251-1271. http://dx.doi.org/10.2307/1913827.

Hawkins, S., Cole, T., Law, C., 2009. Examining the relationship between maternal employment and health behaviours in 5-year-old British children. J. Epidemiol. Commun. H. 63, 999-1004. http://dx.doi.org/10.1136/jech.2008.084590.

Holcomb, W.L., Chaiworapongsa, T., Luke, D.A., Burgdorf, K.D., 2001. An odd measure of risk: use and misuse of the odds ratio. Obstet. Gynecol. 98, 685-688.

Jabs, J., Devine, C.M., Bisogni, C.A., Farrell, T.J., Jastran, M., Wethington, E., 2007. Trying to find the quickest way: employed mothers' constructions of time for 
food. J. Nutr. Educ. Behav. 39, 18-25. http://dx.doi.org/10.1016/ j.jneb.2006.08.011.

Jarman, M., Inskip, H.M., Ntani, G., Cooper, C., Baird, J., Robinson, S.M., Barker, M.E. 2014. Influences on the diet quality of pre-school children: importance of maternal psychological characteristics. Public Health Nutr. 18, 2001-2010. http://dx.doi.org/10.1017/S136898001400250X.

Kendall, G.E., Li, J., 2005. Early childhood socialization and social gradients in adult health: a commentary on Singh-Manoux and Marmot's "Role of socialization in explaining social inequalities in health" (60: 9, 2005, 2129-2133). Soc. Sci. Med. 61, 2272-2276. http://dx.doi.org/10.1016/j.socscimed.2005.08.034.

Kleiner, S., Pavalko, E.K., 2010. Clocking in: the organization of work time and health in the United States. Soc. Forces 88, 1463-1486. http://dx.doi.org/10.1353/ sof.0.0301.

Labour Statistics Division, 2007. The Canadian Labour Market at a Glance. Catalogue no. 71-222-X.

Landivar, L.C., 2015. The gender gap in employment hours: do work-hour regulations matter? Work Employ. Soc. 29, 550-570. http://dx.doi.org/ $10.1177 / 0950017014568139$.

Li, J., O'Sullivan, T., Johnson, S., Stanley, F., Oddy, W., 2011. Maternal work hours in early to middle childhood link to later adolescent diet quality. Public Health Nutr. 15, 1861-1870. http://dx.doi.org/10.1017/S1368980011003053.

Lobstein, T., Jackson-Leach, R., Moodie, M.L., Hall, K.D., Gortmaker, S.L. Swinburn, B.A., James, W.P.T., Wang, Y., McPherson, K., 2015. Child and adolescent obesity: Part of a bigger picture. Lancet 385, 2510-2520. http:// dx.doi.org/10.1016/S0140-6736(14)61746-3.

Marshall, N.L., Barnett, R.C., 1993. Work-family strains and gains among two-earner couples. J. Community Psychol. 21, 64-78.

McNall, L.A., Masuda, A.D., Nicklin, J.M., 2009. Flexible work arrangements, job satisfaction, and turnover intentions: the mediating role of work-to-family enrichment. J. Psychol. 144, 61-81. http://dx.doi.org/10.1080/ 00223980903356073.

Morrissey, T.W., 2013. Trajectories of growth in body mass index across childhood: associations with maternal and paternal employment. Soc. Sci. Med. 95, 60-68. http://dx.doi.org/10.1016/j.socscimed.2012.05.038.

Petersen, T., 2004. Analyzing panel data: fixed- and random-effects models. In: Bryman, A., Hardy, M.A. (Eds.), Handbook of Data Analysis. Sage, London, pp. $331-345$.

Phipps, S.A., Lethbridge, L., Burton, P., 2006. Long-run consequences of parental paid work hours for child overweight status in Canada. Soc. Sci. Med. 62, 977-986.

Speirs, K.E., Leichty, J.M., Wu, C.-F., Strong Kids Research Team, 2014. Sleep, but not other daily routine, mediates the association between maternal employment and BMI for preschool children. Sleep. Med. 15, 1590-1593. http://dx.doi.org/ 10.1016/j.sleep.2014.08.006.

Tuttle, R., Garr, M., 2012. Shift work and work to family fit: does schedule control matter? J. Fam. Econ. Issues 33, 261-271. http://dx.doi.org/10.1007/s10834-0129283-6.

von Hinke Kessler Scholder, S., 2008. Maternal employment and overweight children: does timing matter? Health Econ. 17, 889-906. http://dx.doi.org/ 10.1002/hec.1357.

Watts, A.W., Laska, M.N., Larson, N.I., Neumark-Sztainer, D.R., 2016. Millennials at work: workplace environments of young adults and associations with weight-related health. J. Epidemiol. Commun. H. 70, 65-71. http://dx.doi.org/ 10.1136/jech-2015-205782.

World Health Organization, 2014. Global Status Report on Noncommunicable Diseases 2010. WHO, Geneva.

Ziol-Guest, K.M., Dunifon, R.E., Kalil, A., 2013. Parental employment and children's body weight: mothers, others, and mechanisms. Soc. Sci. Med. 95, 52-59. http://dx.doi.org/10.1016/j.socscimed.2012.09.004. 